\title{
Algunas causas de la mala práctica docente en lenguas extranjeras
}

\author{
Jorge García Mata \\ Escuela Oficial de Idiomas de Málaga
}

Recibido: 26 diciembre 2007 / Aceptado: 13 febrero 2008

ISSN: 1697-7467

\begin{abstract}
It is as important to highlight the features of good foreign language teachers as it is to point out the evident pitfalls of those who are not in that group. Being a professional teacher -in the sense of making one's living from teaching- is far from automatically being a good teacher: this calls for other traits that may be categorized as 'personal', 'methodological' or 'linguistic'. This paper explores these categories and the way they interact with one another according to previous models, and describes how teachers may enter into either positive or negative cycles that severely affect their professional development.
\end{abstract}

Key words: teacher training; responsibility; bad teachers; professionalism; foreign language.

RESUMEN: Tan importante como resaltar los rasgos de los buenos profesores de lengua extranjera resulta señalar los evidentes fallos de aquellos que no pertenecen a tal grupo. Ser un profesional de la enseñanza -en el sentido de ganarse la vida con ésta - no lo convierte a uno automáticamente en un buen profesor: ello requiere otros atributos que pueden categorizarse como 'personales', 'metodológicos' o 'lingüísticos'. Este artículo explora dichas categorías y el modo en que se interrelacionan, según modelos previos, y describe cómo los profesores pueden entrar en ciclos positivos o negativos que afectan seriamente su desarrollo profesional.

Palabras clave: formación del profesorado; responsabilidad; malos profesores; profesionalismo; lengua extranjera.

\section{El mal profesor de lengua extranjera (le)}

El énfasis en los aspectos positivos de cualquier cuestión no obedece solamente a la necesidad de evitar tratamientos potencialmente ofensivos, también responde a razones estratégicas por cuanto se entiende que la presentación del lado positivo de los fenómenos resulta más estimulante, más directa para la consecución de los fines que se persiguen, y, por lo tanto, más persuasiva. No obstante lo anterior, acompañar a la ilustración de las ventajas a obtener una descripción de los defectos a evitar difícilmente puede considerarse contraproducente. La literatura sobre el buen profesor es muy abundante y no agotaré al lector con referencias ya clásicas (para una revisión actualizada del concepto ver, p. ej., Kelly y otros, 2004; Jackson, 2006); el presente trabajo pretende constituir una visión complementaria -quizá menos delicada y diplomática- de algunos aspectos de aquellos estudios positivos y, 
aunque se centra en el análisis de sus correspondientes facetas negativas, pretende contribuir a evitar algunos errores fundamentales para la buena práctica decente de LE, señalándolos y analizándolos de una manera tan clara y directa como sea posible.

\section{Profesionalismo y Profesionalidad en la enseñanza de le}

No conozco ningún estudio de amplio alcance cuyo objetivo sea medir de forma válida y fiable el nivel de buena práctica profesional entre los profesores de LE de Educación Primaria en España. Sospecho que tal investigación, caso de realizarse, arrojaría unos resultados incómodos para las autoridades educativas y provocaría la airada protesta de organizaciones sindicales y de los colectivos afectados, pidiendo inmediatamente la no generalización de tales conclusiones. La realidad es otra: nuestro país ocupa un lugar poco destacado en la comparación con otros países de nuestro entorno en materia de nivel de uso de la población de una LE (ver, p. ej., Bonnet, 2002, para el caso concreto del inglés); cabe suponer que esos resultados desfavorables puedan ser achacados a una variedad de razones, entre las que destacarían las inadecuadas condiciones en que se lleva a cabo esa instrucción y, sobre todo, a una deficiente formación y selección del profesorado (Pedró, 2006). Interesa, por tanto, en el diseño de estrategias apropiadas para corregir estos defectos, contar con unos criterios claros de qué se entiende por mal profesor de LE. Circunscribimos nuestro interés en estas páginas al nivel educativo de Primaria.

La docencia de LE, como la de las demás áreas curriculares en todas las etapas de nuestro sistema educativo, se halla confiada a un cuerpo profesionalizado, compuesto por personas que han obtenido una cualificación universitaria, que desarrollan una labor tipificada y regulada legalmente, cuyos miembros comparten unos intereses laborales (Hargreaves y Goodson, 1996; citado en Hargreaves, 2000), y son objeto de una cierta consideración por parte de otros grupos sociales (Helsby, 1995; citado en Hargraves, 2000). Desgraciadamente, en muchos casos aquí acaba la descripción de estos profesionales, y ello es porque otro atributo de esta profesionalización es la opacidad que envuelve el desarrollo de su labor, ya que como 'profesionales' exigen que se les respete un alto grado de independencia y discrecionalidad en su docencia (Hoyle, 1975; citado en Locke y otros, 2005), convirtiendo su aula en un espacio inaccesible para terceros, donde es virtualmente imposible constatar sus buenas o malas prácticas.

Es posible otra visión de las características de estos colectivos, centrada predominantemente no en cómo son vistos sus miembros por el resto de la sociedad o de qué prerrogativas gozan en virtud de su posición, sino en cómo se consideran ellos mismos y qué exigencias se plantean en relación con el carácter y calidad de sus iniciativas profesionales (Hargreaves y Goodson, 1996; citado en Hargreaves, 2000), y con la responsabilidad de desarrollar y controlar su propio conocimiento y actuación para beneficio de sus alumnos (Webb y otros, 2004). Opuesta a la visión aislacionista de la profesionalidad esbozada más arriba -que consagra, entre otras, la no necesidad de rendir cuentas sobre la propia actuación docente y las razones en que ésta se sustenta-, el concepto de profesionalismo propone una visión abierta de la enseñanza como una actividad reticular que se extiende necesariamente más allá del propio aula (Hoyle, 1975; citado en Locke y otros, 2005). 
Tener como profesión la enseñanza de LE y ser un profesional de la enseñanza de LE son, como se viene sugiriendo, dos conceptos bien distintos; y, aunque ambos son factores necesarios en la delimitación del perfil del profesor, la actual situación de la enseñanza de LE en este país parece mostrar una descompensación evidente a favor de la primera, que analizamos con más detalle en los apartados que siguen.

\section{Componentes del PERfil del PROFESOR DE LE}

Partiendo de la caracterización general del profesor como persona-que enseña (Edge, 2002), Gabrielatos (2002) precisa la del profesor de LE como persona-que enseña-LE, recogiendo esta descripción los tres ejes que estructuran, a su juicio, el perfil de los miembros de este grupo ocupacional: el ámbito personal $(\mathrm{P})$, el espacio metodológico $(\mathrm{M})$, y el terreno del conocimiento y uso de la LE (L). Examinemos, a continuación, qué significa ser un mal profesor según cada uno de estos tres ejes.

\subsection{Deficiencias relacionadas con factores de personalidad ('Es un duro / un blando / un raro / ...')}

Desde el punto de vista del alumnado, la personalidad del profesor puede ser percibida como un factor negativo en la experiencia de aprendizaje de LE por diversos motivos que pueden darse aislados o en combinación, haciendo más o menos desagradable $-\mathrm{y}$, por tanto, en diversos grados infructuosa- dicha experiencia. Entre los factores del ámbito $\mathrm{P}$ mencionados por Gabrielatos (2002), nos parece que destacan por su relevancia las habilidades interpersonales. Cualquier profesor con dificultades para relacionarse con los demás por excesiva timidez, por arrogancia, por escasa empatía, por demasiada tensión, por incapacidad para separar convenientemente las esfera de lo profesional y lo privado, por dificultades para integrarse adecuadamente en un entorno socio-laboral, por obstáculos para la comunicación, por falta de de sentido del humor, etc., introduce en el aula un modo de relación variablemente inadecuado, que los alumnos perciben de manera imprecisa, pero que puede suponer el establecimiento de patrones de relación muy negativos, que impidan o reduzcan muy significativamente el aprendizaje, alienando a los alumnos.

Los desequilibrios en materia de personalidad no son infrecuentes, y su incidencia entre los profesionales de la enseñanza no suele ser debidamente tomada en cuenta ni valorada; si algunos entran en la esfera de lo patológico, muchos son simplemente interpretados como rasgos idiosincrásicos a asumir por el entorno; en cualquier caso, no parece haber respuesta para este problema. Si en todo ejercicio profesional es profundamente deseable que sus miembros muestren una mínima habilidad interpersonal, en la enseñanza de LE esta exigencia es crítica, puesto que se enseña (o se debe enseñar) LE para comunicarse a través de ella, y la comunicación depende de la existencia de canales interpersonales abiertos y fluidos.

Otro aspecto de la personalidad del profesor que puede ser detectado negativamente por los alumnos de LE es la falta de flexibilidad (por ejemplo, ante la necesidad de introducir cambios, ante la diversidad de los alumnos y de sus necesidades, ante las situaciones que requieran desplegar autoridad, etc.). Un profesor inflexible es reconocido inmediatamente -aunque no siempre de forma consciente- por los alumnos como alguien débil e inseguro, 
poco sensible a las condiciones del contexto, e inaccesible: difícilmente la imagen de quien debe inspirar confianza y seguridad en los demás. Muchos profesores confunden la firmeza con la inflexibilidad, y desean mostrar la primera, cuando de lo único que son capaces es de funcionar a través de la segunda: los alumnos distinguen muy fácilmente entre ambas, aunque, es necesario insistir, no siempre conscientemente.

No todos los atributos de la personalidad del profesor capaces de introducir serias dificultades en su desarrollo profesional son directamente observables por los alumnos, y esto los hace más difíciles de detectar y combatir, ya que muchos profesores no son conscientes de ellos y quedan, en consecuencia, ocultos a lo largo de toda una carrera profesional; dentro de la lista de Gabrielatos (2002), hemos elegido para ilustrar estas barreras el bajo nivel de autoconciencia, la escasa competencia reflexiva, y una poco desarrollada percepción de lo que es el aprendizaje de una LE. Como cualquier profesional, el profesor de LE que no reflexiona sobre los contenidos de su especialidad, que no se plantea sistemáticamente sus creencias y principios sobre qué es enseñar y aprender una LE, que no observa, analiza y extrae conclusiones sobre las reacciones de los alumnos a sus iniciativas docentes, que no valora la importancia de una formación continua de calidad, que piensa, en resumen, que no tiene nada que aprender, está irremisiblemente destinado a repetir sus involuntarios aciertos sin potenciarlos, y sus errores sin corregirlos.

\subsection{Deficiencias relacionadas con factores metodológicos ('No sabe dar clase')}

Aparte de preferencias específicas individuales, se puede afirmar que todo alumno de LE aspira a tener la sensación de que aprende lo suficiente, a un ritmo adecuado y con un esfuerzo aceptable; en estas tres parcelas exhibe una influencia decisiva la metodología que decida emplear el profesor. Las posibilidades de insatisfacción por parte del alumnado son diversas: el profesor puede imponer un ritmo demasiado intenso, sin dejar tiempo para asimilar lo tratado; puede mostrarse incapaz de explicar adecuadamente ciertas complejidades, o no concebir maneras alternativas de esclarecerlas; puede presentar los contenidos en secuencias carentes de coherencia para los alumnos o insuficientemente planeadas; no variar el modo de trabajo ni de agrupación de los alumnos; o depender exclusivamente del libro de texto sin apenas introducir otros materiales; o centrarse excesivamente en el tratamiento de ciertas habilidades comunicativas en detrimento de otras -generalmente introduciendo un énfasis desproporcionado en lo escrito sobre lo oral; no revisar cíclicamente lo tratado anteriormente; carecer de una variedad mínima de recursos; proponer una y otra vez el mismo tipo de tarea, o ser éstas de un nivel cognitivo inadecuado, o no proporcionar un equilibrio entre el reto que representan y el apoyo que se presta abocando a los alumnos al fracaso sin reconocer, como profesor, su propia responsabilidad.

Los alumnos no tienen dificultad en reconocer y proclamar que quienes cometen algunas -o muchas- del sucinto catálogo de incompetencias reseñado más arriba son malos profesores. De hecho, los alumnos parecen reservar esta etiqueta casi exclusivamente para los profesores que muestran graves deficiencias metodológicas, de las que son agudamente conscientes a través de sus ideas y creencias implícitas -mucho más articuladas conforme ganan experiencia discente. Infelizmente, otra característica (perteneciente al ámbito P) del mal profesor es su incapacidad para reconocer dicha capacidad crítica de sus alumnos, a los que 
tienden a considerar recipientes vacíos tanto en conocimientos, como experiencia y expectativas. Corre pareja esta errónea apreciación con algunas otras, como la de que enseñar implica necesariamente aprender; que la responsabilidad de la enseñanza-aprendizaje recae únicamente sobre el profesor; que la transmisión es un proceso unívoco desde el profesor al alumno; que no todos los alumnos están capacitados para aprender una LE; que aprender una LE es, en todo caso, algo extraordinariamente difícil y que lo único que él puede hacer como profesor es afianzar los conocimientos formales morfosintácticos y léxicos; y, que en general, si los alumnos no aprenden es porque no quieren o no pueden, no porque él no haga todo lo posible.

\subsection{Deficiencias relacionadas con factores de conocimientos ('No tiene ni idea')}

Es improbable que un alumnado que detecta claras limitaciones en el conocimiento y uso de la LE por parte de su profesor de LE lo etiquete de manera tan poco compasiva, a no ser que dichas dificultades aparezcan acompañadas de otras en uno o ambos de los otros dos ámbitos (P y M). Si no son justificables severas deficiencias por parte de los profesores de LE en ninguno de los tres ejes (P, M o L), menos aún parecen serlo en el tercero (L), por cuanto resulta más inmediato valorar este factor por medio de pruebas previas oportunas. El problema, al menos en nuestro país, parece estribar en que las autoridades educativas y los empresarios del sector de la enseñanza consideran acomodaticiamente que el hecho de que el candidato esté en posesión de cierta cualificación universitaria les exonera de toda responsabilidad de comprobar las excelencias que dicha titulación teóricamente proclama.

El hecho incontestable es que un número significativo de profesores de LE en ejercicio en nuestro país no tiene el nivel de competencia mínimo necesario, sobre todo oral, en el uso de esa LE, un hecho particular y dolorosamente claro en la Educación Primaria. Aquel profesor que no use regularmente la LE como vehículo de comunicación en el aula, bien porque carezca de la competencia para hacerlo, bien porque no se encuentre lo suficientemente seguro, bien porque considere -erróneamente- que sus alumnos no podrían beneficiarse de esa iniciativa por falta de nivel, está cometiendo algo parecido a un fraude, ya que priva deliberadamente a sus alumnos de la única exposición -ya de por sí bastante reducida- a un uso cercano a lo comunicativo de la LE, y les impide interiorizar la posibilidad y utilidad de ésta como vehículo de comunicación. Estamos, pues, en presencia de un mal profesor de LE sin paliativos; como también lo es aquél que carece de los conocimientos necesarios sobre el funcionamiento de la LE para explicar adecuadamente una duda, poner ejemplos claros y variados, introducir el tratamiento de aspectos culturales relevantes a través de la LE, y hacer un uso de ella que constituya un modelo adecuado para los alumnos.

Los alumnos perdonarán en distinta medida las deficiencias anteriores, pero ello no resta su importancia, sobre todo porque, objetivamente, son las menos difíciles de subsanar a medio plazo si los responsables de selección de profesorado de LE insistieran en la comprobación directa de las competencias deseables en los candidatos, sobre todo orales, a través de pruebas especializadas y referidas al contexto del aula, así como comprobando regularmente que la LE es el instrumento habitual de comunicación en el aula de LE. 


\section{Triángulos de profesionalismo y CírCulos de mala pRÁCtica en la ENSEÑANZA DE LE}

Un somero repaso de la bibliografía sobre el profesionalismo docente nos revela un sorprendente interés por la trigonometría. Tal vez nuestra visión espacial nos haga especialmente atractivo visualizar fenómenos a lo largo de tres ejes, creando un espacio tridimensional a lo largo, ancho y alto del cual situar los valores de las categorías que definen tales fenómenos. Alternativamente, los tres ejes pueden no abrir el espacio sino limitar el plano intersecándose de dos en dos para formar un triángulo; resulta interesante que ésta sea la representación elegida por algunos autores para el concepto de profesionalismo docente, por cuanto apunta a lo cerrado y acotado, al menos visualmente. De manera general, Locke y otros (2005) definen la parcela del profesionalismo docente como un triángulo cuyos lados son la medida respectiva de la autonomía, del altruismo y del conocimiento alcanzados por un profesor concreto (Fig. 1a).

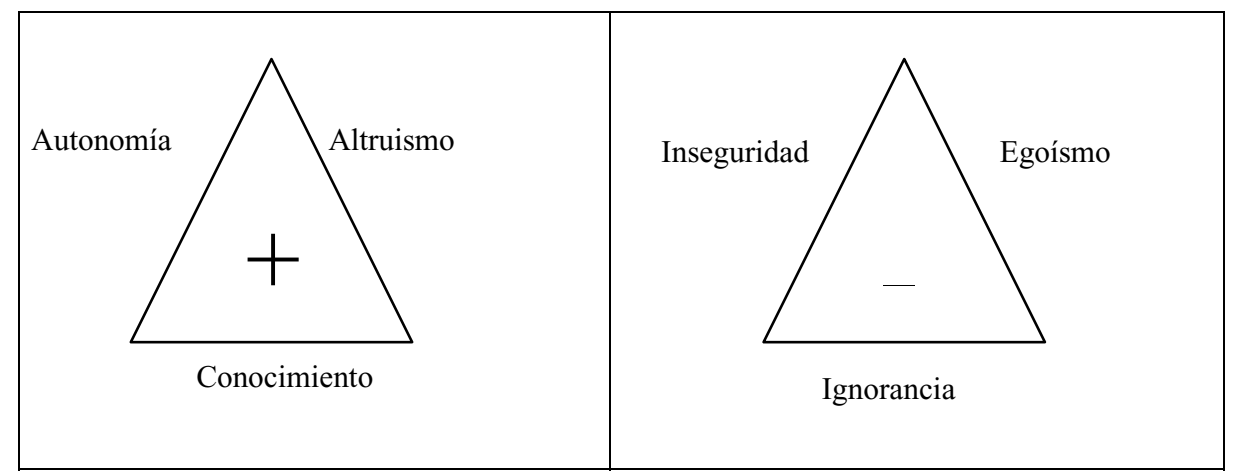

Figura 1a. Profesionalismo positivo.

Figura 1b. Profesionalismo negativo.

Desde el punto de vista del profesionalismo, el mal profesor (Fig. 1b) se caracteriza por una mayor o menor inseguridad y necesidad de dependencia de una 'autoridad' (esto es, la 'tradición', el libro de texto, la normativa en sus aspectos formales, etc.); en segundo lugar, por una variable dosis de egoísmo que le impulsa a anteponer constante y sistemáticamente sus intereses a los de los alumnos y a los de la institución educativa en la que están insertos; $\mathrm{y}$, finalmente, muestran diversos grados de desconocimiento sobre su propia materia.

Si el triángulo de la figura $1 \mathrm{~b}$ representa una visión general del mal profesor, Gabrielatos (2002) propone un triángulo específico para el profesor de LE (Fig. 1c), que transformamos en negativo (Fig. 1d) para caracterizar al mal profesor de LE. 
Algunas causas de la mala práctica docente ...

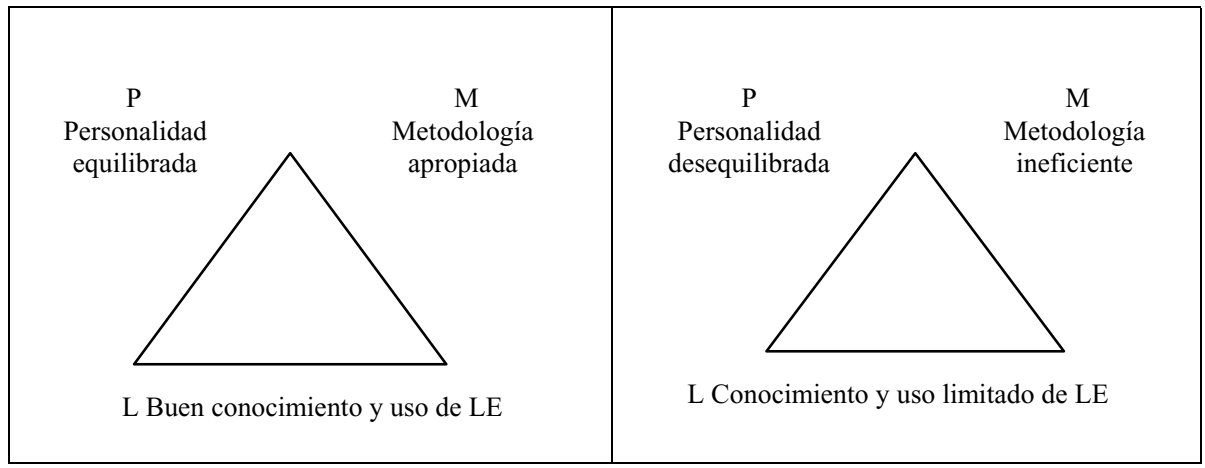

Figura 1c. El profesionalismo positivo en LE. Figura 1d. El profesionalismo negativo en LE.

El valor de la propuesta de Gabrielatos (2002) (Fig. 1c) reside en que representa la interacción de los tres factores $(\mathrm{P}, \mathrm{M}$ y L) a través del área total del triángulo que delimitan. Así, el área máxima del triángulo corresponde con una distribución equilátera, de manera que cuanto más desarrollados y de manera más equilibrada los tres factores, se optimiza el área del triángulo y describen a un mejor profesor. Alternativamente, la reducción exagerada del valor de uno sólo de los factores (ver ejemplo en Fig. 1e) impone formas generales isósceles o escalenas al triángulo, con la consiguiente pérdida de área.

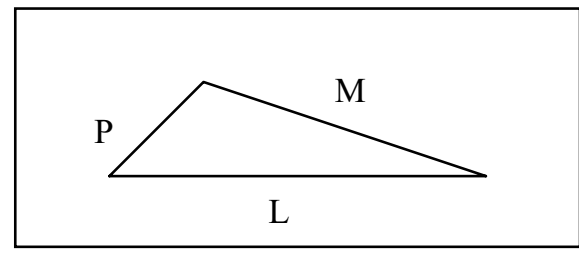

Figura 1e. Ejemplo de perfil descompensado por atrofia del factor P.

Extraemos como consecuencia del símil triangular de Gabrielatos (2002) que la atrofia de uno sólo de los factores que influyen en el profesionalismo de un profesor tiene insoslayablemente serias consecuencias en el profesionalismo global de éste y que, por tanto, la sobreabundancia de cualquier otro factor no puede compensar la desventaja que supone la mengua de uno dado. Como profesores de LE, no podemos -de acuerdo con esta visióncontentarnos con ostentar un conocimiento exhaustivo del funcionamiento de la LE, o un excelente nivel en su uso si, a la vez, carecemos de una metodología eficaz y eficiente para ayudar a aprenderla, y/o adolecemos de ciertos desequilibrios de personalidad que nos dificultan la comunicación, empatía, etc., con nuestros alumnos.

Tal vez un rasgo propio de una personalidad desequilibrada (p. ej., inseguridad, en la Fig. 1b), propia del mal profesor, haga que se interprete la situación descrita en la Figura 1d -y lo dicho en el párrafo anterior-, como inmutable ('yo soy así y no puedo cambiar'); de hecho, merece la pena plantearse cómo puede funcionar este mecanismo de perpetuación de 
la falta de profesionalismo y de la mala práctica; nos valemos para ello del conocido modelo de aprendizaje experiencial (Kolb, 1981), que puede representarse así:

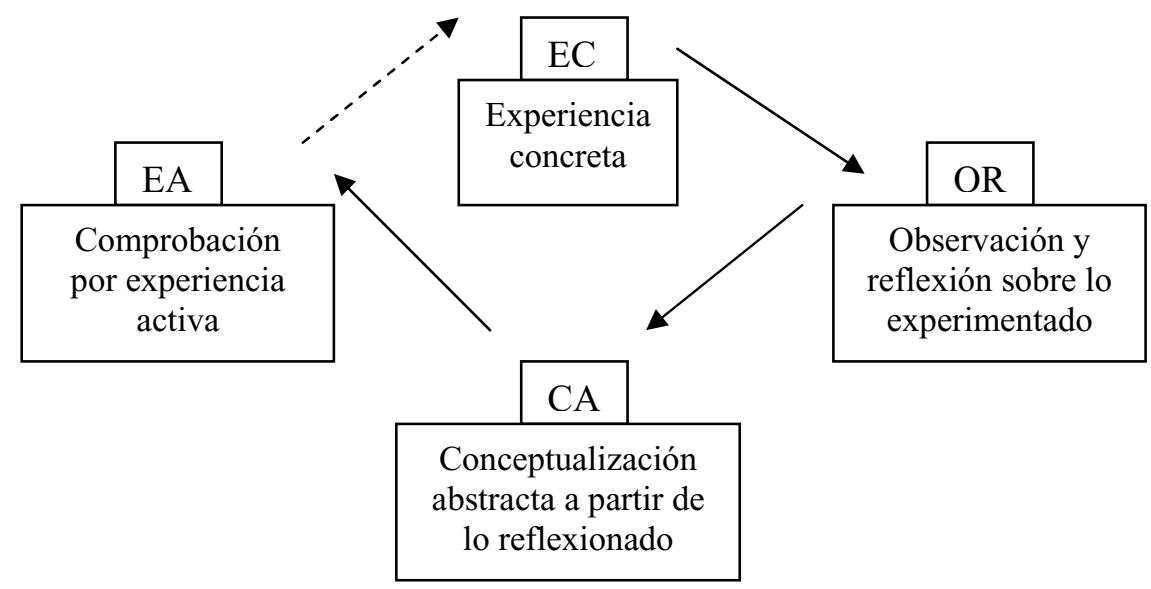

Figura 2a. El ciclo de aprendizaje experiencial (Kolb, 1981).

Al igual que con los triángulos estudiados más arriba, también en el ciclo experiencial podríamos proponer aplicaciones de éste para ilustrar procesos acumulativos de mejora o deterioro de la práctica y del profesionalismo en la enseñanza de LE. Nos centramos aquí por falta de espacio- en los ciclos negativos en que se embarcan los malos profesores. Veamos, en primer lugar, uno de muchos posibles ciclos generales que podrían explicar que un profesor de LE se encierre cada vez más en una visión negativa de su función profesional, que probablemente podría conducirle en última instancia a un profundo desinterés por su labor y, en consecuencia, a un desempeño deficiente de ésta. Representamos las distintas fases del ciclo en términos de pensamientos verbalizados por el profesor.

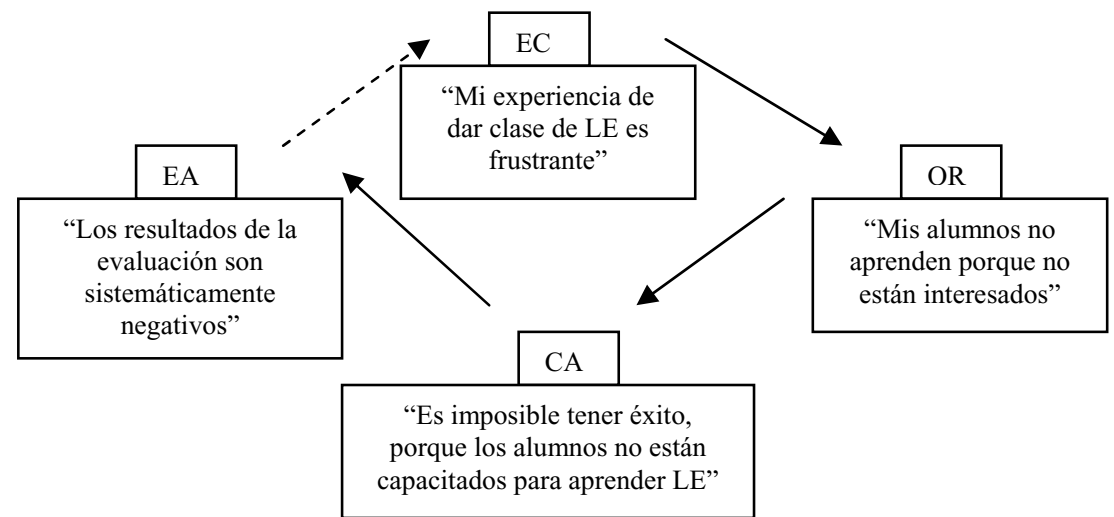

Figura 2b. Un ciclo general de profesionalismo negativo. 
Puede argumentarse que los ciclos de este tipo se originan por una limitada capacidad del profesor para analizar y reflexionar sobre la experiencia -0 , simplemente, por falta de voluntad para embarcarse en un proceso sincero de autoexamen y análisis de las causas de la situación-, lo que induce a un escrutinio superficial y generalmente erróneo en la fase OR del ciclo, que arrastra a una generación de hipótesis inadecuada en la CA, que tiende a confirmarse como una 'profecía autocumplida' en la fase EA; lo que, a su vez, prepara el terreno para la vivencia negativa reforzada en la fase EC del siguiente ciclo.

A modo de ilustración concreta -y para cerrar este apartado- del funcionamiento negativo recién descrito, se desarrollo un ciclo negativo que puede ayudar a explicar una de las realidades de aula más representativa de la mala práctica en la enseñanza de LE: el uso exageradamente limitado, o incluso ausente, de la LE como código de comunicación cotidiano en el aula:

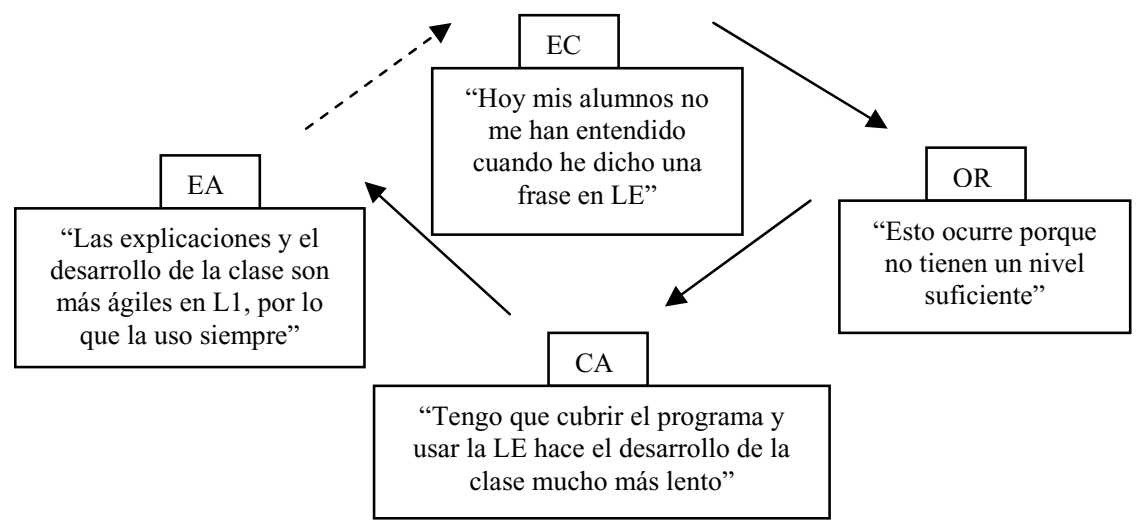

Figura 2c. Ciclo ilustrativo de falta de uso de LE en el aula.

\section{RESPONSABILIDAD Y COLABORACIÓN: DOS SOLUCIONES DEMASIADO LEJANAS}

Un posible origen para los ciclos negativos de mala práctica puede hallarse en la ausencia del ejercicio de responsabilidad y de colaboración por parte de los malos profesores de LE. En rigor, no puede culparse directamente a los profesores de este estado de cosas; como profesores vivimos insertos en un sistema educativo cuyos valores reales subyacentes -a diferencia de aquellos que promueve la normativa legal al efecto- reflejan fielmente los de la sociedad a la que sirve, en la que no son precisamente la responsabilidad individual y el espíritu de colaboración los valores predominantes. No obstante, una visión amplia del profesionalismo se basa precisamente en los conceptos de responsabilidad y de colaboración.

\subsection{La responsabilidad en el profesionalismo}

Conviene deslindar dos connotaciones del término responsabilidad. En primer lugar, ser responsable se interpreta como aquel rasgo de la personalidad individual en virtud del cual 
el individuo que lo muestra se encuentra moralmente sujeto a actuar de una determinada manera en virtud de unos compromisos contraídos consigo mismo; esta sería la visión interna de la responsabilidad (expresada en inglés como responsibility), que implica fiabilidad. Pero ser responsable también puede implicar la situación en que se encuentra un individuo que debe rendir cuentas de sus decisiones y de su actuación ante otros, y afrontar las consecuencias derivadas de aquéllas, bien en términos de estímulo bien de censura (nos referimos aquí al término anglosajón accountability). Ambas interpretaciones son necesarias en la definición de un adecuado profesionalismo, y ambas se articulan sobre la exigencia (interna o externa) y sobre la existencia de un escrutinio del que se derivan consecuencias. Sin desdeñar la importancia de la responsabilidad interna, resulta más interesante centrar esta breve indagación en la externa, por cuanto concita el tratamiento de un elemento imprescindible en el buen desarrollo de organizaciones sociales y, dentro de éstas, de las profesionales.

En efecto, para Locke y otros (2005) la falta de profesionalismo se relaciona con la tensión existente entre los factores de autonomía, altruismo y conocimiento (Fig. 1a) y el concepto de responsabilidad externa. Así, cuando el sistema no exige regularmente una rendición de cuentas al profesor de LE por el uso de su autonomía, se corre el riesgo de que ese profesor no haga uso efectivo de ella y permanezca indefinidamente en un estado de dependencia de la tradición heredada en materia de enseñanza de LE; similarmente, la tensión entre altruismo y responsabilidad puede conducir a la perpetuación de actitudes egoístas e insolidarias en el profesorado de LE; finalmente, cuando el sistema renuncia a pedir cuentas al profesor de LE sobre su conocimiento y capacidad de uso de la LE, la consecuencia inevitable es la relajación de muchos cuyos conocimientos y capacidad de uso de la LE fueron aceptables en un momento dado, o la imposibilidad efectiva de detectar regularmente insuficiencias preexistentes y actuar frente a ellas proponiendo acciones de formación complementaria.

\subsection{La dificultad para la colaboración}

La colaboración, como la responsabilidad -en su connotación de rendición de cuentas-, es un acto mediante el cual nos sometemos al escrutinio por parte de otros de nuestra competencia profesional; si bien la segunda resulta más explícita y suele venir acompañada de las correspondientes recompensas o recriminaciones, la primera constituye un acto mucho más íntimo en el que se desvelan nuestras creencias, principios y procedimientos ante nuestros colegas que, de esta forma, acceden a un conocimiento de nosotros como profesionales que podría en último término - sobre todo si la colaboración no es genuina- permitirles establecer comparaciones de las que se podría salir malparado.

Por el contrario, la colaboración bien entendida depende críticamente de la confianza (Troman, 2000). La desconfianza, que se basa en sentimientos internos de inseguridad, suspicacia, inadecuación, etc., hace imposible entrar en relaciones de colaboración con colegas a quien la padece, por razones que se han ilustrado en el párrafo anterior. La confianza mutua -que no puede establecerse en ausencia de unos mínimos niveles de autoconfianza en cada uno de los intervinientes- no sólo favorece la colaboración sino también la autonomía y la creatividad (Alexander, 1989; citado en Troman, 2000) que son, a su vez, manifestaciones de la autoconfianza, estableciéndose así un ciclo positivo de reforzamiento mutuo entre desarrollo de la autoconfianza y desarrollo de la confianza mutua entre colegas. El principal obstáculo para que este deseable ciclo se instaure es la existencia de una colaboración forzada, 
opuesta a otra libre y genuina (Webb y otros, 2004). En principio, puede argumentarse que el punto de entrada en el ciclo positivo de desarrollo de la autoconfianza y de la confianza mutua es un mínimo de desarrollo de la primera en los individuos que tiene intención de colaborar (Gambetta, 1980; citado en Troman, 2000) -que, cabe suponer, se deberá en las etapas iniciales del desarrollo profesional a factores tanto de la personalidad del profesor como a contextuales, y, dentro de estos últimos, a los modelos de profesor a los que haya estado expuesto a lo largo de la propia experiencia discente.

De no existir el mínimo nivel de autoconfianza antes aludido sería imposible entrar en colaboración genuina y surgirían entonces dos posibilidades: renunciar al contacto profesional con colegas, o entrar en una colaboración forzada, lo que a su vez permitiría dos posibilidades: que la iniciativa para establecer colaboración viniese de uno mismo a través de la presión ejercida por la cultura de colaboración imperante en el contexto -cultura ante la que el profesor podría no encontrarse cómodo, pero a la que no desearía enfrentarse-, o que la institución o sistema educativo regule a través de normativa la obligación de desarrollar actividades colaborativas con los compañeros de centro. Ninguna de estas situaciones puede llevar fácilmente a una colaboración sincera, abierta y productiva, ya que no se da el requisito previo de la confianza, al ser imposible que se establezca un cierto grado de predictabilidad entre los cooperantes forzados (Nias y otros, 1989; citado en Troman, 2000), en virtud de la cual los intervinientes saben qué pueden esperar de los demás, y que su interacción se desarrollará dentro de unos parámetros siempre positivos y mutuamente reforzadores, carentes de intenciones formalmente evaluadoras, sino centradas en el aspecto formador y del desarrollo profesional.

Es posible recurrir a la estructura de ciclos negativos desarrollada en la sección anterior para analizar el funcionamiento del rechazo en las situaciones de colaboración forzada.

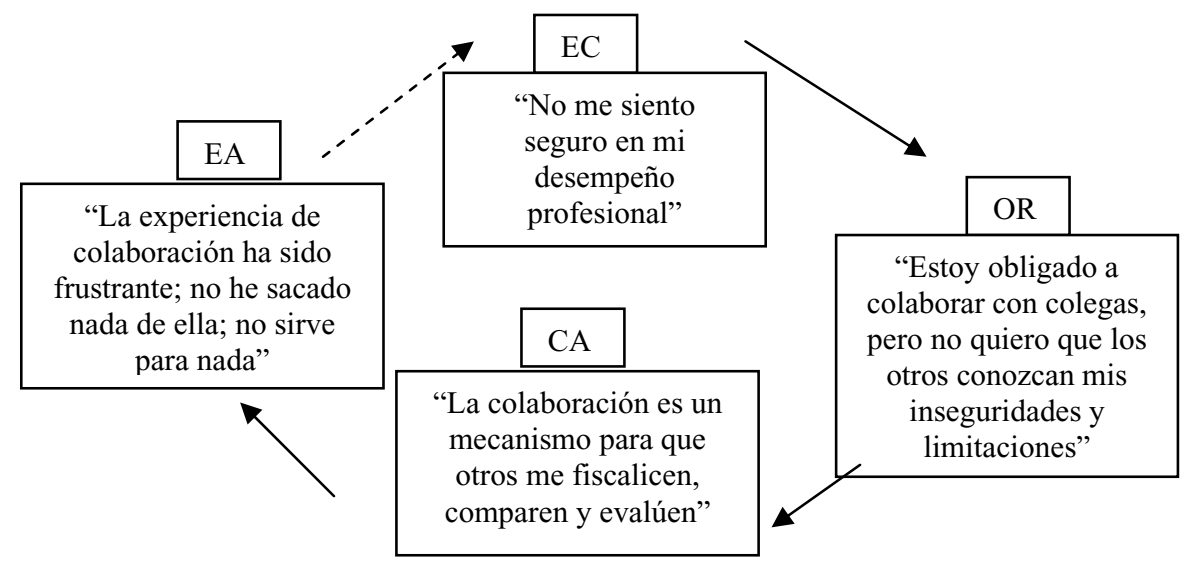

Figura 3a. Ciclo negativo general ante colaboración forzada. 
Como todos los ciclos negativos que definen la falta de profesionalismo y la mala práctica, el de la Figura 3a es un ciclo de alienación que supone la introducción de un factor de agravamiento de la situación negativa y un bloqueo de sus posibilidades de mejora. Al igual que en los otros ciclos negativos tratados anteriormente, el de la Figura 3 a tiene su origen en un defecto de la fase OR; en este caso, el vicio se relaciona con una deficiente asunción de la autonomía por parte del profesor alienado, que muestra una baja capacidad crítica para tomar decisiones propias, con renuncia a la libertad a través de la adhesión acrítica a decisiones tomadas por otros; esta actitud lleva en último término a unas mal entendidas autonomía y libertad de acción, que toma la forma del fatal ciclo siguiente:

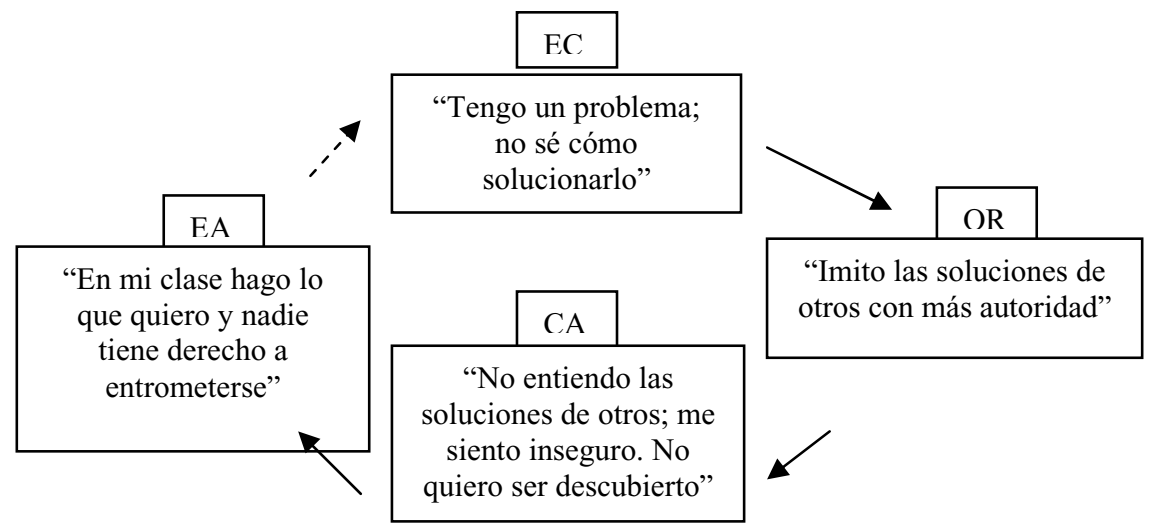

Figura 3b. Ciclo negativo de autoconfianza.

Como muestra el ciclo de la Figura 3b, la reacción defensiva de ciertos profesores que apelan a la autonomía, libertad e independencia en el desarrollo de su labor para bloquear los intentos de sus entornos de poner en marcha iniciativas colaborativas, de docencia compartida, de investigación y observación cooperativa en el aula, de compartir materiales, recursos, ideas, etc., tiene su origen precisamente en un déficit por su parte de autonomía, de libertad y de independencia; en cierta forma, apelan a la presunción de goce de tales atributos para evitar tener que asumirlos, y viven consecuentemente bajo el peso de tal decisión.

\section{Conclusión}

Resulta a un tiempo urgente y desproporcionada la tarea de evaluar la práctica de los profesionales encargados de la docencia de LE en nuestro país. Es urgente por cuanto el coste económico y social que asume nuestra sociedad ante el alarmantemente bajo nivel de uso comunicativo de LE entre la población es enorme, y seguirá creciendo en un mundo cada vez más interconectado, donde las decisiones de inversión externa tienen muy en cuenta las capacidades y formación de los futuros empleados; también a nivel personal, puesto que una parte importante de la población se verá privada de posibilidades reales de participación en mercados laborales internacionales, y acceso a otras culturas. 
Confiamos en que lo expuesto en este trabajo haya permitido al lector apreciar la inmensa dificultad de la tarea de reducir las malas prácticas docentes en las aulas de LE en nuestro país, y ello por varias razones: en primer lugar, porque la bondad profesional docente es un concepto multifacético y complejo; en segundo lugar, porque afecta a un número elevado de profesionales en ejercicio; en tercer lugar, porque son previsibles reacciones adversas y defensivas; y, finalmente, porque el ejercicio de la profesión docente -como cualquier otro- está inscrito en los parámetros y valores de una sociedad escasamente familiarizada con alternativas, que considera normales y aceptables -en algún caso con resignación- ciertos comportamientos y metodologías en el aula de LE, y que, en consecuencia, no demanda cambios.

No es esperable una variación sustancial a corto plazo en la calidad de las prácticas profesionales en las aulas de LE en nuestro país, pero sí es exigible que las autoridades educativas desarrollen un modelo simple y claro de profesorado de LE de calidad, con unas competencias reducidas, concretas y esenciales; resulta asimismo perentorio, que esas autoridades asuman la responsabilidad de evaluar y vigilar que esos mínimos estándares de buena práctica se cumplan; la aceptación de esos patrones y su puesta en práctica no puede quedar a la voluntad individual de los profesores.

\section{REFERENCIAS BIBLIOGRÁFICAS}

Alexander, J. (1989), «The Modern Reconstruction of Classical Thought», en T. Parsons (ed.) Theoretical Logic in Sociology. Londres: Routledge and Kegan Paul.

Bonnet, G. (Ed.) (2002). The assessment of pupil's skills in English in eight European countries. The European Network of Policy Makers for the Evaluation of Education Systems.

Edge, J. (2002). «A Choice of Horizons for People Who Teach». Conferencia Plenaria en el XXIII Annual TESOL Greece Convention. Atenas.

Gabrielatos, C. (2002). «The shape of the Language Teacher», en A. Pulverness, (ed.) IATEFL York Conference Selections. York: IATEFL: 75-78.

Gambetta, D. (1988). «Can we trust trust?», en D. Gambetta (ed.) Trust: making and breaking cooperative relationships. Oxford: Basil Blackwell.

Hargreaves, A. (2000). Four Ages of Professionalism and Professional Learning. Teachers and Teaching: History and Practice, 6 (2): 151-182.

Hargreaves, A. \& Goodson, I. (1996). «Teachers professional lives: aspirations and actualities», en I. Goodson \& A, Hargreaves (eds.) Teachers' Professional Lives. Londres: Falmer Press.

Helsby, G. (1995) «Teachers' construction of professionalism in England in the 1990s» en Journal of Education for Teaching, 21, 3: 317- 332.

Hoyle, E. (1975) «Professionality, professionalism and control in teaching», en V. Houghton, R. McHugh \& M. Colin (eds.) Management in education: the management of organizations and individuals. Londres: Ward Lock Educational, 314-320.

Jackson, M. (2006). «Serving Time»: the relationship of good and bad teaching», en Quality Assurance In Education, 14, 4: 385-397.

Kelly, M., Grenfell, M., Allan, R., Kriza, C. y Mcevoy, W. (2004). European Profile for Language Teacher Education - A Frame of Reference. Strasburg: European Commission. Directorate General for Education and Culture. 
Kolb, D. A. (1981) «Learning styles and disciplinary differences», en A. W. Chickering (ed.) The Modern American College. San Francisco: Jossey-Bass.

Locke, T., Vulliamy, G., Webb, R. y Hill, M. (2005). «Being a 'professional' primary school teacher at the beginning of the 21st century: a comparative analysis of primary teacher professionalism in New Zealand and England», en Journal of Education Policy, 20, 5: $555-581$

Nias, J. (1989). Primary Teachers Talking: a study of teaching as work. Londres: Routledge.

Pedró, F. (2006). «Un diagnóstico de la situación del profesorado en España desde una perspectiva comparativa», en Revista de Educación, 340: 243-264.

Troman, G. (2000). "Teacher Stress in the Low-Trust Society», en British Journal of Sociology of Education, 21, 3: 331-353.

Webb, R., Vulliamy, G., Hämäläinen, S., Sarja, A., Kimonen, E. y Nevalainen, R. (2004). «A comparative analysis of primary teacher professionalism in England and Finland», en Comparative Education, 40, 1: 83-107. 